\title{
CRISPR/Cas9 assisted gene targeting efficiently inhibits bovine herpesvirus- 1 replication
}

\section{Pallavi Deol}

Indian Veterinary Research Institute

\section{Sonalika Mahajan}

Indian Veterinary Research Institute

\section{Sukdeb Nandi}

Indian Veterinary Research Institute

Vishal Chander

Indian Veterinary Research Institute

Ashwini R. Chaple

Indian Veterinary Research Institute

Saima M Ganie

Indian Veterinary Research Institute

Bindu S.

Indian Veterinary Research Institute

Ravikant Agrawal

Indian Veterinary Research Institute

Karam Pal Singh

Indian Veterinary Research Institute

Vivek kumar gupta

Indian Veterinary Research Institute

Raj kumar Singh

Indian Veterinary Research Institute

Gaurav Kumar Sharma ( $\nabla$ gaurvet@gmail.com )

ICAR-Indian Veterinary Research Institute: Indian Veterinary Research Institute https://orcid.org/00000002-9996-9422

\section{Research Article}

Keywords: Bovine herpesvirus-1 (BHV-1), CRISPR/Cas9, Antiviral strategy, Gene editing

Posted Date: May 13th, 2021

DOI: https://doi.org/10.21203/rs.3.rs-473984/v1 
License: (c) (i) This work is licensed under a Creative Commons Attribution 4.0 International License. Read Full License 


\section{CRISPR/Cas9 assisted gene targeting efficiently inhibits bovine herpesvirus-1 replication}

Pallavi Deol ${ }^{1}$, Sonalika Mahajan ${ }^{1 *}$, Sukdeb Nandi ${ }^{1}$, Vishal Chander ${ }^{1}$, Ashwini R. Chaple ${ }^{1}$, Saima M. Ganie ${ }^{1}$, Bindu $S^{1}$, Ravi Kant Agrawal ${ }^{1}$, Vivek Kumar Gupta ${ }^{2}$, Karam Pal Singh ${ }^{1}$, Raj Kumar Singh ${ }^{3}$, Gaurav Kumar Sharma ${ }^{1 *}$

${ }^{1}$ Indian Council of Agriculture Research (ICAR)- Indian Veterinary Research Institute (IVRI), Bareilly 243122, India

${ }^{2}$ Director, Indian Council of Agriculture Research (ICAR)- National Research Centre on Pig (NRCP), Guwahati, 781131, India

${ }^{3}$ Ex-Director, Indian Council of Agriculture Research (ICAR)- Indian Veterinary Research Institute (IVRI), Bareilly 243122, India

*Corresponding e-mail: gaurvet@gmail.com; sonalikam1@gmail.com

\section{Abstract}

Clustered Regularly Interspaced Short Palindromic Repeats (CRISPR)/ CRISPR associated protein (cas) are now being accepted as a highly specific method of gene editing. Among many other applications, CRISPR/cas has an immense potential to be used as antivirals. In this study, we successfully demonstrated CRISPR/Cas9 mediated inhibition of Bovine Herpes virus -1 (BHV-1) replication. BHV-1 causes economically important diseases in bovines with establishment of latency. Six essential genes and one non-essential gene of BHV-1 were targeted to assess the impact on virus replication. Inhibition of UL52, circ, and UL27 genes showed promising results, whereas the other three genes US6, UL18, and UL34 resulted in lower level of inhibition. Non-specific gene editing in host and virus was in-silico evaluated and was demonstrated by inhibition of virus induced apoptosis. Successful editing of one viral non-essential gene without any alterations in virus replication demonstrated the potential of CRISPR/Cas9 in replicating viral genome. Complete abrogation of virus replication was observed transiently ( 24 hours post-transfection/hpt) when transfected with short lived in-vitro transcribed sgRNAs. Whereas, under constant expression of sgRNAs through plasmid, complete inhibition of virus replication was observed till $\sim 72$ hours postinfection. Complete inhibition of replication was also observed with in-vitro transcribed sgRNA when booster dose of sgRNA was trasnfected at 24hpt. It has been speculated that 
constant expression with plasmid based delivery may result in off-target activity which can be ruled out with short lived in-vitro transcribed sgRNA.

\section{Keywords}

Bovine herpesvirus-1 (BHV-1), CRISPR/Cas9, Antiviral strategy, Gene editing

Infectious diseases are one of the biggest threats to the livestock and poultry industry around the globe. Arguably, diseases of viral aetiology are considered crucial among all infectious diseases as they spread rapidly across the boundaries and results in serious socio-economic and public health consequences [1]. Large-scale mortality, drastic reduction in farm output, zoonotic potential, and trade embargo are some of the major concerns related to the viral diseases [2]. Control and eradication of viral pathogens are essential towards achieving global food security [3]. With a lack of effective antiviral drugs, control efforts rely mainly on vaccination and biosecurity measures. Some diseases have been controlled and eradicated whereas many important viral diseases are still endemic and continue to cause huge economic losses despite several vaccination-based efforts. Bovine herpesvirus-1 (BHV-1) is one such example that is endemic in many countries. BHV-1 belongs to the Alphaherpesvirinae subfamily of the Herpesviridae and cause diseases in domestic and wild cattle. During the primary infection BHV-1 causes abortion, infectious bovine rhinotracheitis (IBR), and infectious pustular vulvovaginitis (IPVV), after which the cattle become a latent carrier [4]. This virus establishes latency mainly in ganglionic neurons where it expresses only a few viral genes and remains inside the host without being noticed by the host's immune response and such latent carriers are the main source of disease dissemination [5-7]. The available vaccines prevent the clinical impact of the disease but not virus infection and latency establishment [4]. Currently, no therapeutics is available for BHV-1 infected/carrier animals.

Lately, CRISPR/Cas9 gene-editing technology has emerged as a powerful tool for RNAguided specific genome modifications, which has the potential to develop new generation therapeutics. CRISPR/Cas9 system has been tested for its therapeutic potential (invitro and in-vivo) for various viruses (reviewed in [8]), either through direct disruption of the viral genome or by hampering the host factors helping in the virus replication [8]. To explore 
a similar strategy for BHV-1, the present study was designed to evaluate the efficiency of the CRISPR/Cas9 system to limit BHV-1 replication.

The essential and non-essential genes required for in-vitro replication of BHV-1 were selected as described by Robinson and co-workers [9]. Six essential genes spread across the three temporal expression zones viz. immediate-early (IE), early (E), and late (L) genes were targeted to achieve maximum inhibition of replication. Glycoprotein E (gE) gene of BHV-1 which is not essential for in-vitro replication was also targeted with CRISPR/Cas9 to evaluate specific genome editing and effects on virus replication. The selected genes and their functions are detailed in table 1. BHV-1 subtype 1.1 was revived in Madin-Darby Bovine Kidney (MDBK) cells maintained in DMEM supplemented with 10\% fetal bovine serum and antibiotics. Cells were observed for cytopathic effects (CPE), the virus was titrated using the end-point dilution method [10] and stored at $-80^{\circ} \mathrm{C}$ till further use.

The single-guide RNA (sgRNA) targets for each selected gene, were designed using the CRISPR RNA guided engineered nucleases (RGENs) tool (http://www.rgenome.net/casdesigner/) and the ones with the highest score for specificity (least/no off-targets) within bovine and BHV-1 genome were selected (table 1). To further ensure the specificity, insilico analysis of sgRNA targets was performed using the web server of Cas-OFFinder (http://www.rgenome.net/cas-offinder/). The primers for the synthesis of the sgRNA DNA template for each selected gene were synthesized commercially (Eurofins, India). sgRNA DNA templates were PCR assembled and confirmed by 2\% agarose gel electrophoresis (AGE). The confirmed templates were in-vitro transcribed to sgRNA, using a commercially available kit (GeneArt precision gRNA synthesis kit, Invitrogen) as per the recommended protocol.

MDBK cells were seeded in 24 well plate and at 60-80\% confluency, cells were infected with BHV-1 (1 MOI). 3-4 hours post-infection (hpi), 125 ng of in-vitro synthesized sgRNA was complexed with 625ng of Cas9 enzyme (Invitrogen) to form RNP complex and transfected into BHV-1 infected MDBK cells using CRISPRMAX transfection reagent as per the manufacturer's recommendations. Virus and mock-infected cell controls were included and the plates were regularly observed for the appearance of characteristic CPE. 
Along with RNP-based expression, the sgRNA target sequences were also expressed via U6 promoter-driven PX459 vector, following the protocol described by Ran and co-workers [11]. Briefly, sgRNA target sequences were cloned in PX459 vector, transformed in the DH5 $\alpha$ strain of E. coli. and the plasmids were extracted using an endotoxin-free plasmid extraction kit (Qiagen plasmid midiprep kit). Similar to RNP based expression method, the MDBK cells were seeded and transfected with plasmids (500ng/well in 24 well plate) cloned with each sgRNA target using p3000 reagent (Thermo, USA) as per manufacturer's instructions. For plasmid-based transfection, BHV-1 infection (1MOI) was given 12-14 hours posttransfection (hpt).

BHV-1 infected and mock-infected cells were harvested in triplicates at 06, 12, 24 and $48 \mathrm{hpt}$ for RNP transfected cells and at 72 hpi for plasmid transfected cells. Virus quantification was performed by endpoint dilution method of titration [11]. Plaque-assay was performed for virus quantification and comparison of plaque morphologies of wild type (WT) and CRISPR targeted BHV-1, as described by Baer and Hall [12]. Viral DNA load was quantified by realtime PCR assay using SYBR chemistry. Specific forward primer (TGTGGACCTAAACCTCACGGT) and reverse primer (GTAGTCGAGCAGACCCGTGTC) were synthesized for quantification using plasmid DNA standards as described elsewhere [13]. In order to ascertain the inhibition of virus replication, virus induced apoptosis [15] was estimated using terminal deoxynucleotidyl transferase dUTP nick end labelling (TUNEL) assay (Promega, USA) as per the manufacturer's instructions.

T7 endonuclease assay was performed to evaluate gene editing in BHV-1 that was targeted for a non-essential gene (gE), using commercially available kit (GeneArt Genome Cleavage detection kit, Thermo, USA) as per the recommendations. Forward primer (FP: CGTGTGTCTTGGTTTCTGCG) and reverse primer (RP: GAAGACCGTGTCGACCGAAG) were designed to amplify CRISPR targeted region to confirm the insertion/deletion. Bhattacharya and Meir, [14] demonstrated that CRISPR/Cas9 induced insertions/deletions can also be detected by high density agarose gel electrophoresis. Therefore, the amplified (used FP and RP) target gene was also resolved on high density (5\%) agarose gel. 
Characteristic CPE for BHV-1 appeared after 12hpi and completed by 48hpi (Fig. 1a-d). The mock infected cells did not show any significant changes till 60hpi. Standard growth curve for virus replication was prepared with the data obtained from virus titration and real-time PCR. The RNP-based experiment exhibited significant reduction in CPE up to 24 hpt (Fig. 1e-g). However, complete CPE was observed at 48hpt (Fig. 1h) which indicated that the RNP complex successfully inhibited the virus replication for 24hpt but not beyond. Contrarily, the CPE in non- essential gene treated BHV-1 (Fig. 1i-l) approximated with WT BHV-1. To confirm this observation, percentage inhibition (PI) of replication in CRISPR/Cas9 treated virus relative to un-treated virus (WT) was calculated, by the end-point dilution method of titration, and the findings corroborated the visual changes. More than $60 \%$ inhibition (relative to BHV-1 controls) was observed at 24hpt in BHV-1 treated with sgRNA against essential genes (Fig. 2a-g). Our experiment revealed that targeting UL52, circ, and UL27 genes of BHV-1 abrogated the virus replication most effectively (98-99.9\%) (Fig. 2a-c), whereas targeting US6, UL18, and UL34, genes resulted in comparatively lower inhibition (68-92\%) (Fig. 2e-g). Furthermore, to completely abrogate the virus replication through RNP based platform, two refinements were tried. At first, all the selected essential genes of BHV-1 were simultaneously targeted but only marginal improvement in inhibition of virus replication was achieved with RNP-based multiple targeting over single gene targeting (Fig. 2d). Secondly, in addition to single dose of RNP complex at 6 hpi, a booster dose was given at 24hpt. Complete abrogation of BHV-1 replication was observed when treated with second dose of RNP. Constant expression of sgRNA through vector-based system against essential genes of BHV-1 also completely inhibited the virus replication till 72hpi.

On the other hand, statistically insignificant ( $\mathrm{p}>0.05)$ inhibition was observed in the BHV-1 treated with sgRNA targeting a non-essential (gE) gene (Fig. 2h), which indicates no harmful effects of the components of CRISPR/cas9 system. Viral gene editing was successfully demonstrated by T7 endonuclease assay which resulted in three amplicons (one; parental and two; cleaved products) in the virus population confirming the presence of mutant as well as WT BHV-1 (Fig. 3c). Similar findings were obtained when PCR amplicons were resolved in high concentration ( $>5 \%$ ) agarose gel electrophoresis that showed multiple PCR amplicons in CRISPR targeted gE amplified products (mutants/MT) which were absent in the untreated WT BHV-1 (Fig. 3b). 
Plaque assay analysis (Fig. 4a-d) revealed that BHV-1 targeted for essential genes produced very few plaques (Fig. 4c) whereas non-essential (gE) gene targeted BHV-1 produced plaques that were smaller in size (Fig. 4d) as compared to WT BHV-1 plaques (Fig. 4b).

Inhibition of virus replication was also demonstrated by TUNEL assay where virus induced apoptosis (Fig. 5b) was reduced in the essential gene targeted BHV-1 (Fig. 5c). On the other hand, negligible changes were observed for a non-essential gene targeted BHV-1 (Fig. 5d).

Over the past few years, specific gene editing by CRISPR/Cas9 has exemplified the possibility for the development of new generation antivirals. In a very short span of time many researchers have demonstrated its use as a potential antiviral strategy in several viruses including hepatitis $\mathrm{C}$ virus, human immunodeficiency virus, herpesviruses (herpes simplex virus-1, Epstein-Barr virus, pseudorabies virus), hepatitis B virus, human papillomavirus, etc [16-20]. In this study, we demonstrated the antiviral potential of CRISPR-Cas9 against BHV1. It was observed that BHV-1 replication is inhibited by a minimum of $60 \%$ on targeting any one of the six essential genes evaluated in this study. BHV-1 genes namely UL52, circ, and UL27 were shown as potential targets to impair BHV-1 replication (Fig. 2a-c). Among all the targeted essential genes, UL27 (encodes for glycoprotein B/ gB) could be the most potential single target because it starts one of the earliest contacts with the host cell, since it acts as a ligand for attachment receptors and also has a role in the fusion of virus envelope with the host cell membrane [4]. We also observed that the RNP complex resulted in significant inhibition only up to 24hpt, and the virus replication again peeked after 24hpt (Fig 2a-g). The plausible reason behind incomplete abrogation could be the limited half-life of the Cas9 enzyme which is approximately 20hrs [21]. Complete abrogation of virus replication has been shown for other viruses using vector-based sgRNA/Cas9 delivery system [16-19]. Complete abrogation of BHV-1 using a vector-based delivery system against all the six genes. Although plasmid-based delivery showed promising results in the inhibition of virus replication, but it is speculated that with plasmid system, there are chances of integration in host genome and persistent activity of Cas9 enzyme might lead to non-specific gene editing in virus or host genome [22]. Therefore, RNP platform with a short half-life should be preferred for the development of antivirals. Consequently, we attempted the delivery of the second dose of sgRNA/Cas9 after 24hpt and complete viral replication abrogation was 
observed, that was similar to the vector-based delivery system. Non-specific effects of sgRNA/Cas9 based gene editing on virus replication were evaluated by keeping one control (gE gene) which is not essential for virus replication in the experiment. No significant drop in virus titer was observed after targeting gE (Fig 2h). Successful gE gene editing was achieved by CRISPR-Cas9 (Fig 3b). The mutation(s) was confirmed by T7 endonucleaseassay indicating the specific nature of genome editing by CRISPR/Cas9 (Fig 3c). Off-target activities of the designed sgRNAs were evaluated in-silico and by sequencing the amplified top five off-targets in the host genome wherein we did not find any changes within the amplified regions. Although CRISPR/Cas9 mediated gene editing is highly specific in nature, confirming minimum/no non-specificity is of utmost importance for the development of antivirals, to ensure safety [8].

It is concluded that BHV-1 replication can be inhibited using CRISPR/Cas9 based gene editing. Although constant expression of sgRNA through vector-based system resulted in complete abrogation of replication over RNP-based platform for a longer duration, but the short half-life of the RNP complex has the advantage of eliminating the chances of off-target activity, which is the most crucial concern for developing new generation antivirals. Hence, multiple dosing of short-lived sgRNAs/Cas9 complex may be attempted for complete inhibition of virus with minimum or no off-target activity. However, further studies including host genome-wide screening to detect non-specificity would be required to confirm the specific antiviral potential of CRISPR/cas9 before it is evaluated in the laboratory animals.

\section{Declarations}

Funding: Indian Council of Agricultural Research (ICAR) provided necessary funding under institute funded project. Indian Council of Medical Research (ICMR) provided a fellowship for the Ph.D. candidate.

Conflict of Interest/Competing Interests: All authors declare that there is no conflict of interest concerning funding or any other.

Availability of data and material: Data generated during the study is available from the corresponding author on reasonable request. 
Code availability: Not applicable.

Authors' Contribution Statement: GKS, RKS, VKG, and SM designed the work. GKS, RKA, and PD conducted the experiments and wrote the manuscript. SN, RKA, and VC contributed to the reagents, kits, and proofread the manuscript. ARC, BS, KPS, and SMG helped in cell culture and molecular work. All authors approved the manuscript.

Ethics approval: This article does not involve any studies with human participants or animals by any of the authors.

Acknowledgments: The authors are thankful to the Indian Council of Agricultural Research (ICAR), Government of India, and Department of Animal Husbandry, Dairying, and Fisheries for providing the necessary facility for this work. The authors are thankful to the Indian Council of Medical Research (ICMR).

1. OIE (2021) Infections and infestations in force in 2021. https://www.oie.int/en/animal-health-in-the-world/oie-listed-diseases-2021/. Accessed 01 March 2021

2. Rich KM, Perry BD (2011) The economic and poverty impacts of animal diseases in developing countries: new roles, new demands for economics and epidemiology. Prev Vet Med. 101:133-47. https://doi: 10.1016/j.prevetmed.2010.08.002

3. Espinosa R, Tago D, Treich N (2020) Infectious diseases and meat production. Environ Resour Econ 76:1019-44

4. Muylkens B, Thiry J, Kirten P, Schynts F, Thiry E (2007) Bovine herpesvirus 1 infection and infectious bovine rhinotracheitis. Vet Res 38:181-209. https://doi: 10.1051/vetres:2006059

5. Winkler MT, Doster A, Jones C 2000 Persistence and reactivation of bovine herpesvirus 1 in the tonsils of latently infected calves. J Virol 74:5337-46. doi: 10.1128/jvi.74.11.5337-5346.2000

6. Thiry E, Saliki J, Bublot M, Pastoret PP (1987) Reactivation of infectious bovine rhinotracheitis virus by transport. Comp Immunol Microbiol Infect dis. 10:59-63. doi: 10.1016/0147-9571(87)90041-5 
7. Jones C (2016) Latency of bovine herpesvirus 1 (BoHV-1) in sensory neurons. Herpesviridae. Sep 7:237. https://10.5772/63750

8. Lee C. CRISPR/Cas9-based antiviral strategy: current status and the potential challenge (2019) Molecules 24:1349. https:// 10.3390/molecules.24071349

9. Robinson KE, Meers J, Gravel JL, McCarthy FM, Mahony TJ (2008) The essential and non-essential genes of Bovine herpesvirus 1. J Gen Virol89:2851-63. https://doi: 10.1099/vir.0.2008/002501-0

10. Reed LJ and Muench H (1938). A simple method of estimating fifty per cent endpoints. Am $\quad$ J Epidemiol 27:493-497. https://doi.org/10.1093/oxfordjournals.aje.a118408

11. Ran FA, Hsu PD, Wright J, Agarwala V, Scott DA, Zhang F (2013) Genome engineering using the CRISPR-Cas9 system. Nat Protoc 8:2281-308. https://doi.org/10.1038/nprot.2013.143

12. Baer A, Kehn-Hall K (2014) Viral concentration determination through plaque assays: using traditional and novel overlay systems. J vis exp 93. 10.3791/52065

13. Abril C, Engels M, Liman A, Hilbe M, Albini S, Franchini M, Suter M, Ackermann M (2004) Both viral and host factors contribute to neurovirulence of bovine herpesviruses 1 and 5 in interferon receptor-deficient mice. J Virol 78:3644-53. https://10.1128/JVI.78.7.3644-3653.2004

14. Bhattacharya D, Van Meir EG (2019) A simple genotyping method to detect small CRISPR-Cas9 induced indels by agarose gel electrophoresis. Sci Rep 9:1-7

15. Xu X, Zhang K, Huang Y, Ding L, Chen G, Zhang H, Tong D (2012) Bovine herpes virus type 1 induces apoptosis through Fas-dependent and mitochondria-controlled manner in Madin-Darby bovine kidney cells. Virol J. 9:1-1

16. Van Diemen FR, Lebbink RJ 2017 CRISPR/Cas9, a powerful tool to target human herpesviruses. Cell Microbiol19:e12694

17. Van Diemen FR, Kruse EM, Hooykaas MJ, Bruggeling CE, Schürch AC, van Ham PM, Imhof SM, Nijhuis M, Wiertz EJ, Lebbink RJ (2016) CRISPR/Cas9-mediated genome editing of herpesviruses limits productive and latent infections. PLoSPathog12:e1005701. doi: https://10.1371/journal.ppat.1005701 
18. Roehm PC, Shekarabi M, Wollebo HS, Bellizzi A, He L, Salkind J, Khalili K (2016) Inhibition of HSV-1 replication by gene editing strategy. Sci Rep 6:1-1. https://doi.org/10.1038/srep23146

19. Dong C, Qu L, Wang H, Wei L, Dong Y, Xiong S (2015) Targeting hepatitis B virus cccDNA by CRISPR/Cas9 nuclease efficiently inhibits viral replication. Antiviral Res 118:110-7. https://10.1016/j.antiviral.2015.03.015

20. Tang YD, Liu JT, Wang TY, Sun MX, Tian ZJ, Cai XH (2017) CRISPR/Cas9mediated multiple single guide RNAs potently abrogate pseudorabies virus replication. Arch Virol. https://162:3881-6. https://10.1007/s00705-017-3553-4

21. Kim S, Kim D, Cho SW, Kim J, Kim JS (2014) Highly efficient RNA-guided genome editing in human cells via delivery of purified Cas9 ribonucleoproteins. Genome res. https: //24:1012-9. 10.1101/gr.171322.113

22. Wu Y, Yang L, Chang T, Kandeel F, Yee JK. A small molecule-controlled Cas9 repressible system. Mol Ther Nucleic Acids 19:922-32. https://doi.org/10.1016/j.omtn.2019.12.026

23. Schröder C, Linde G, Fehler F, Keil GM (1997) From essential to beneficial: glycoprotein D loses importance for replication of bovine herpesvirus 1 in cell culture. J Virol. https://71:25-33. 10.1128/JVI.71.1.25-33.1997

24. Haanes EJ, Thomsen DR, Martin S, Homa FL, Lowery DE (1995) The bovine herpesvirus 1 maturational proteinase and scaffold proteins can substitute for the homologous herpes simplex virus type 1 proteins in the formation of hybrid type B capsids. J Virol. https: //10.1128/JVI.69.11.7375-7379.1995

25. Pokhriyal M, Ratta B, Yadav BS, Kumar A, Saxena M, Verma OP, Sharma B (2018) Three newly identified Immediate Early Genes of Bovine herpesvirus 1 lack the characteristic Octamer binding motif-1. Sci Rep 30:1-8. https://doi.org/10.1038/s41598-018-29490-8

26. Fraefel C, Ackermann M, Schwyzer M (1994) Identification of the bovine herpesvirus 1 circ protein, a myristylated and virion-associated polypeptide which is not essential for virus replication in cell culture. J Virol68:8082-8. https://doi.org/10.1099/jgv.0.000823 
Fig 1 MDBK cells were infected with BHV-1 (1MOI), 3-4 hrs prior to sgRNA/Cas9 complex transfection. a-d represents the BHV-1 controls (no gene-targeted) observed at $6,12,24$, and 48 hpt for changes in CPE. e-h shows inhibition of CPE by sgRNA/Cas9 complex targeted against six essential genes (UL52+ Circ+ UL27+US6+UL18+UL34). CPE changes in non-essential gene (gE) targeted BHV$1(\mathrm{i}-\mathrm{I})$

Fig 2 CRISPR/Cas9 targeting of essential genes inhibits BHV-1 replication (a-g). BHV-1 infected MDBK cells were transfected with sgRNAs against the indicated genes (a-h). To assess the percentage of BHV-1 replication inhibition (relative to virus control), viruses were collected at $6,12,24$, and 48 hours post-transfection (hpt) and serially diluted 10-fold (10-1 to 10-8). Each dilution was replicated six times and the virus titer (TCID50/ml) was calculated using Reed and Muench method and (h) shows the BHV-1 replication inhibition for a non-essential gene

Fig 3 Detection of $g E$ gene mutants. (A) The sequence of PCR amplified region targeting gE gene of BHV-1, including sgRNA target (highlighted yellow). Primer (FP and RP) positions used for amplification are shown (orange arrows) which amplifies an expected 381 bpamplicon of wild type (WT) virus (B) Gel image showing WT and mutant (MT) amplicons, $\mathrm{M}$ denotes 100bp DNA marker. CRISPR/Cas9 targeted region was PCR amplified using primers FP/RP and the PCR products were size separated by high concentration (5\%) agarose gel electrophoresis for $80 \mathrm{~min}$. Two amplicons of $381 \mathrm{bp}$ (WT) and 400bp mutant (MT)were seen. Heteroduplex bands were visible in mutant BHV-1. (C) The same set of primers (FP/RP) were used to amplify $381 \mathrm{bp}$ amplicon, after denaturation/re-annealing, samples were treated with 
and T7 endonuclease enzyme and were run on a 2\% agarose gel. Three bands: 381 (parental/WT), 300bp, and $80 \mathrm{bp}$ cleaved bands were seen in the mutated BHV-1 whereas a single band (381 bp) was visible in WT BHV-1

Fig 4 Reduction in BHV-1 plaques with inhibition of essential genes by CRISPRcas9 at 24hpi. (a) Non-infected cell control (b) un-treated BHV-1 (WT) in 10-2 dilution (c) BHV-1 treated with pool of sgRNAs against UL52, Circ, UL27, US6, UL18 and UL34 in 10-2 dilution (d) BHV-1 treated with sgRNA against non-essential gene $(\mathrm{gE})$ in 10-2 dilution

Fig 5 Inhibition of BHV-1 replication resulted in reduction of virus induced apoptosis in MDBK cells by inhibiting the essential genes of BHV-1. Apoptosis-positive cells were detected by terminal deoxynucleotidyl transferase dUTP nick end labeling (TUNEL) assay (uptaking brown color)(a) MDBK cell control (b) BHVI-1 (WT) control (c) CRISPR targeted essential genes (d) CRISPR targeted non-essential (gE) gene 

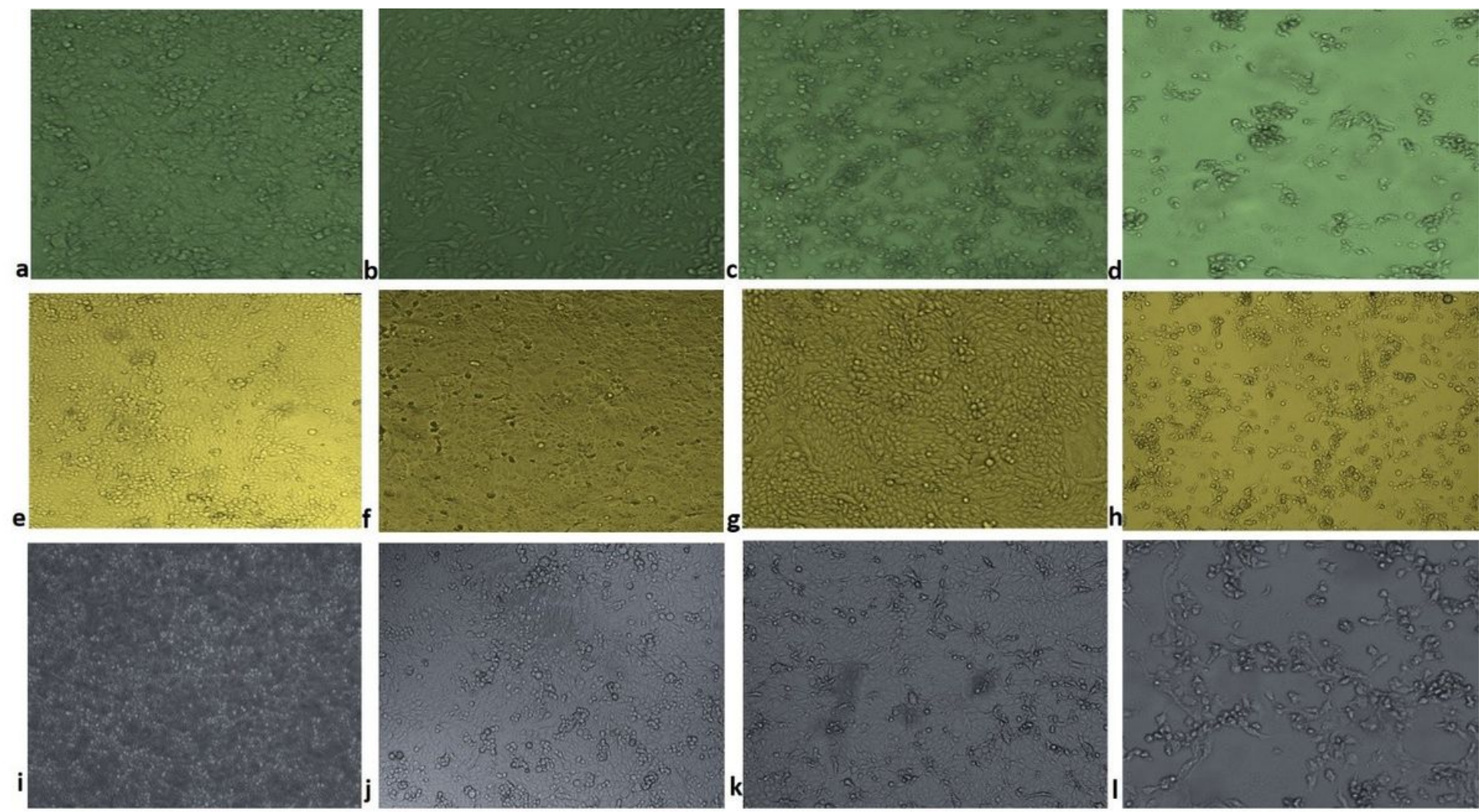

\section{Figure 1}

MDBK cells were infected with BHV-1 (1MOI), 3-4 hrs prior to sgRNA/Cas9 complex transfection. a-d represents the BHV-1 controls (no gene-targeted) observed at 6, 12, 24, and $48 \mathrm{hpt}$ for changes in CPE. e-h shows inhibition of CPE by sgRNA/Cas9 complex targeted against six essential genes (UL52+ Circ+ UL27+US6+UL18+UL34). CPE changes in non-essential gene $(\mathrm{gE})$ targeted $\mathrm{BHV}-1(\mathrm{i}-\mathrm{I})$

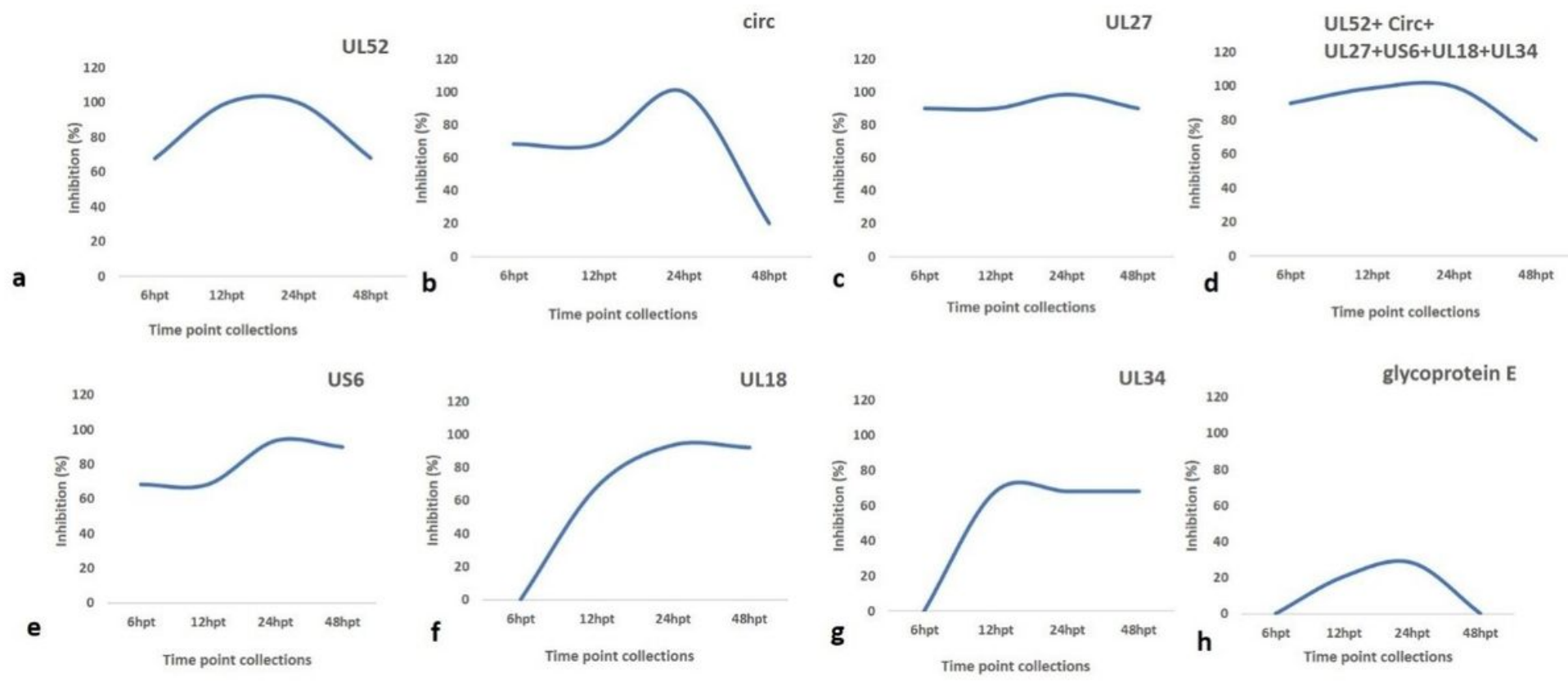




\section{Figure 2}

CRISPR/Cas9 targeting of essential genes inhibits BHV-1 replication (a-g). BHV-1 infected MDBK cells were transfected with sgRNAs against the indicated genes (a-h). To assess the percentage of BHV-1 replication inhibition (relative to virus control), viruses were collected at 6, 12, 24, and 48 hours posttransfection (hpt) and serially diluted 10-fold (10-1 to 10-8). Each dilution was replicated six times and the virus titer (TCID50/ml) was calculated using Reed and Muench method and (h) shows the BHV-1 replication inhibition for a non-essential gene

FP
CGTGTGTCTTGGTTTCTGCGCGGCGGGCGGGG
TGGGAGCGGGCAAGGCGGAGGAAGACCGGGG
GCAGGAGCTGCGTGGAGGGCGGAGCCGTTGAG
CGGCCCGACGCCGCCGGTTGTAAATGGGTC
TCGCGCGGCTCGTGGTTCCACACCGCGCCGGAG
AACCAGCGCGAGCTTCGCTGCGTGTGTCCCGCG
AGCTGCGTTCCGGGGAACGGCGCACGCGAGAG
GGTTCGAAAAGGGCATTTGGCAATGCAACCCAC
CGCGCCGCCCCGGCGGCGGTTGCTGCCGCTGCT
GCTGCCGCAGTTATTGCTTTTCGGGCTGATGGCC
GAGGCCAAGCCCGCGACCGAAACCCCGGGCTC
GGCTTCGGTCGACACGGTCTTC
RP

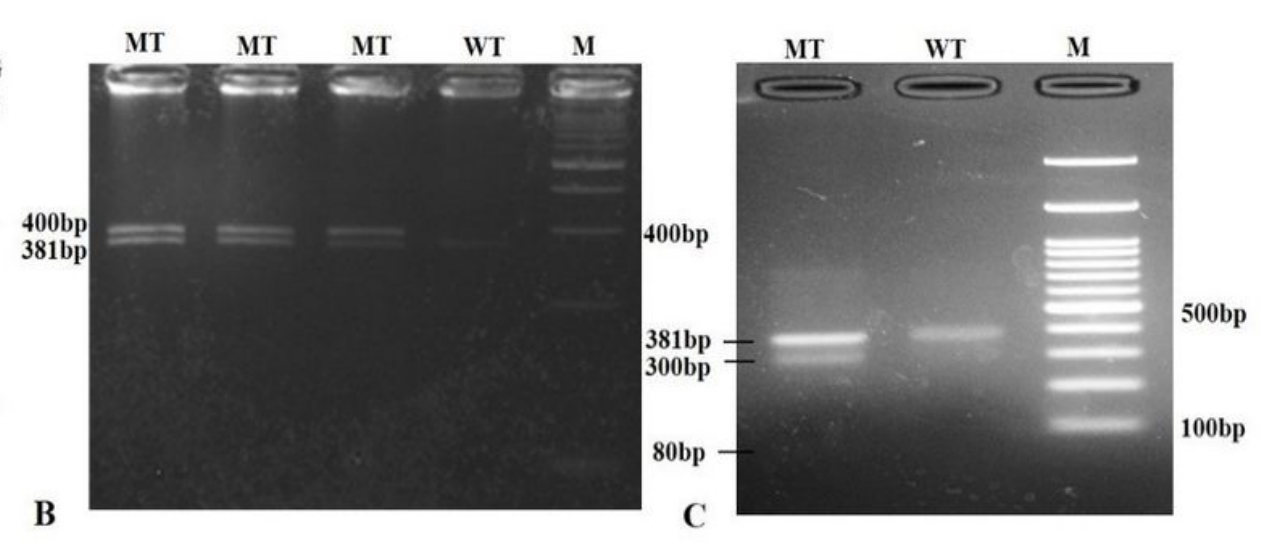

\section{Figure 3}

Detection of gE gene mutants. (A) The sequence of PCR amplified region targeting gE gene of BHV-1, including sgRNA target (highlighted yellow). Primer (FP and RP) positions used for amplification are shown (orange arrows) which amplifies an expected 381 bpamplicon of wild type (WT) virus (B) Gel image showing WT and mutant (MT) amplicons, M denotes 100bp DNA marker. CRISPR/Cas9 targeted region was PCR amplified using primers FP/RP and the PCR products were size separated by high concentration (5\%) agarose gel electrophoresis for $80 \mathrm{~min}$. Two amplicons of $381 \mathrm{bp}$ (WT) and 400bp mutant (MT)were seen. Heteroduplex bands were visible in mutant BHV-1. (C) The same set of primers (FP/RP) were used to amplify $381 \mathrm{bp}$ amplicon, after denaturation/re-annealing, samples were treated with and T7 endonuclease enzyme and were run on a $2 \%$ agarose gel. Three bands: 381 (parental/WT), $300 \mathrm{bp}$, and $80 \mathrm{bp}$ cleaved bands were seen in the mutated BHV-1 whereas a single band (381 bp) was visible in WT BHV-1 


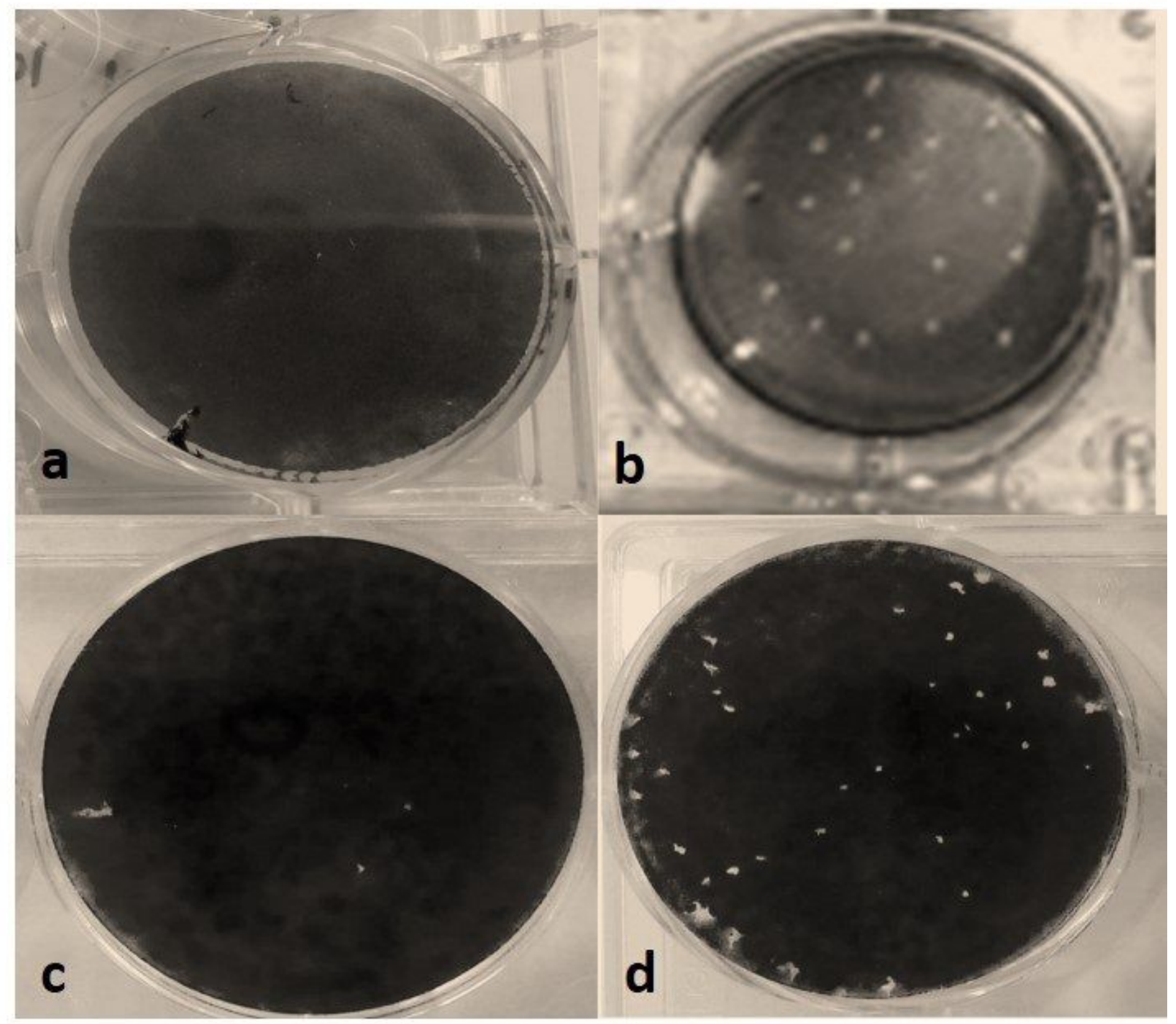

\section{Figure 4}

Reduction in BHV-1 plaques with inhibition of essential genes by CRISPRcas 9 at 24hpi. (a) Non-infected cell control (b) un-treated BHV-1 (WT) in 10-2 dilution (c) BHV-1 treated with pool of sgRNAs against UL52, Circ, UL27, US6, UL18 and UL34 in 10-2 dilution (d) BHV-1 treated with sgRNA against non-essential gene (gE) in 10-2 dilution 


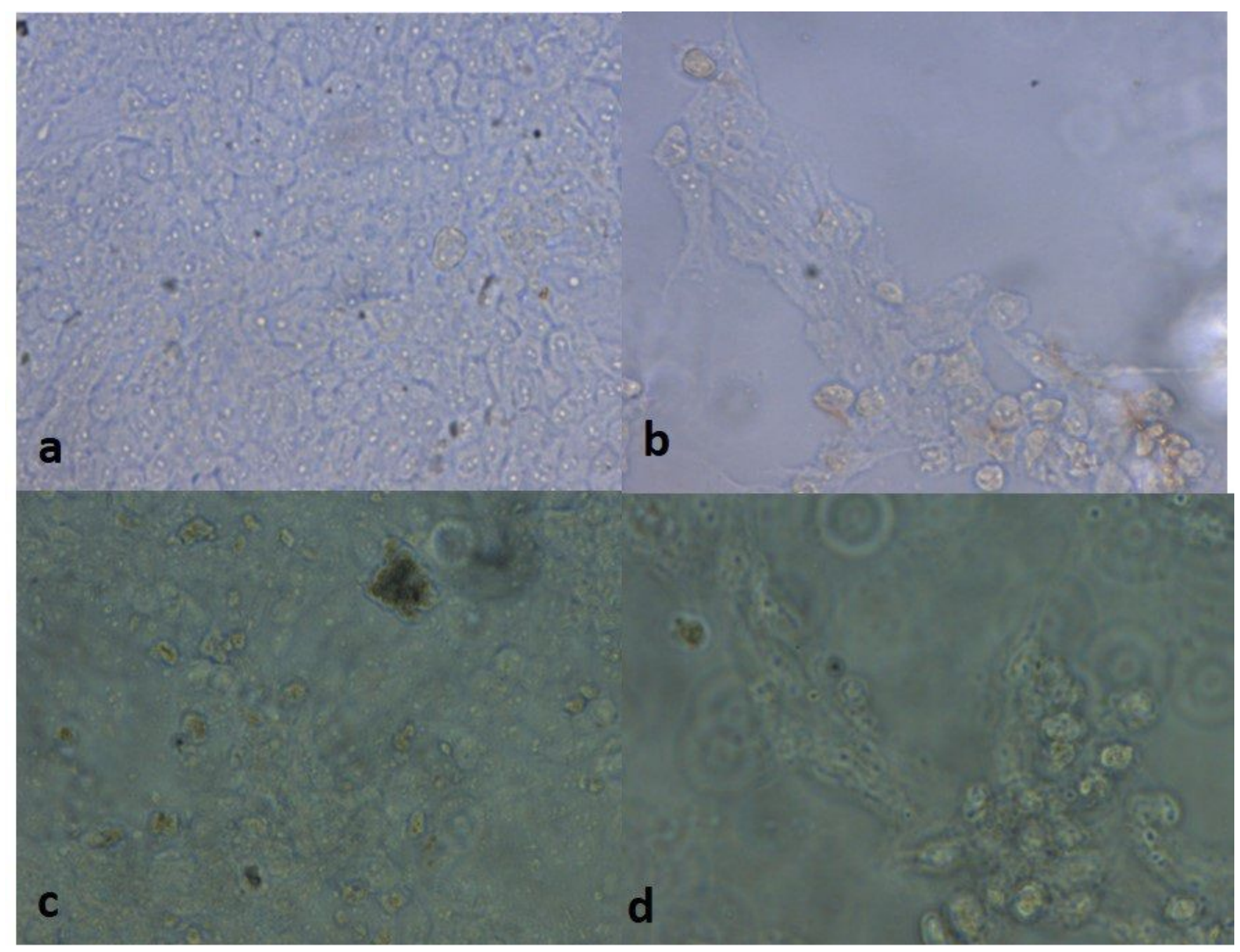

\section{Figure 5}

Inhibition of BHV-1 replication resulted in reduction of virus induced apoptosis in MDBK cells by inhibiting the essential genes of BHV-1. Apoptosis-positive cells were detected by terminal deoxynucleotidyl transferase dUTP nick end labeling (TUNEL) assay (uptaking brown color)(a) MDBK cell control (b) BHVI1 (WT) control (c) CRISPR targeted essential genes (d) CRISPR targeted non-essential (gE) gene

\section{Supplementary Files}

This is a list of supplementary files associated with this preprint. Click to download.

- Table.pdf 